\title{
Evaluation of the expression of stem cell markers in human breast cancer reveals a correlation with clinical progression and metastatic disease in ductal carcinoma
}

\author{
TRACEY AMANDA MARTIN and WEN GUO JIANG \\ Metastasis and Angiogenesis Research Group, Institute of Cancer and Genetics, \\ Cardiff School of Medicine, Cardiff University, Cardiff CF14 4XN, UK
}

Received September 3, 2013; Accepted October 2, 2013

DOI: 10.3892/or.2013.2813

\begin{abstract}
The tumor stem cell theory could explain how patients with metastatic disease show clinical relapse several months after starting treatment due to the survival of a small group of cells with unique characteristics. We examined the distribution and expression of a panel of stem cell markers in human breast cancer primary tumors. Human breast tissues were processed for immunohistochemistry, and RNA was extracted for analysis by quantitative-PCR Immunohistochemical assay revealed that CD44 was strongly expressed in background endothelia and epithelia. CD133 expression was lost in tumor-associated endothelial cells Conversely, CD49b was strongly stained in the tumors, associated vessels and ducts but was weakly stained in the background epithelia. q-PCR analysis revealed that CD44 and PSCA were reduced in patients with poor outcome (metastatic disease and death from breast cancer), with a marked reduction in ductal carcinoma, particularly with metastasis to bone although these did not reach significant difference. CD133 was significantly reduced in patients with metastatic disease and was also significantly reduced in patients with ductal carcinoma/bone metastasis. Conversely, CD49F was increased in patients with a poor outcome and those with ductal cancer and bone metastases. This is the first study to determine the distribution and expression pattern of these stem cell markers in human breast cancer. There was a significant association between loss of expression and metastatic disease in patients with breast cancer. Such differential expression may play a part in breast cancer disease progression, and suggests that the current stem cell theory may not hold true for all cancer types.
\end{abstract}

Correspondence to: Dr Tracey Amanda Martin, Metastasis and Angiogenesis Research Group, Institute of Cancer and Genetics, School of Medicine, Cardiff University, Heath Park, Cardiff CF14 4XN, UK

E-mail: martinta1@cf.ac.uk

Key words: breast cancer, stem cell marker, prognosis

\section{Introduction}

Breast cancer is by far the most frequently diagnosed cancer in woman; more than a million women are diagnosed with breast cancer every year. More than half the cases are in industrialized countries, with approximately 426,900 new cases of breast cancer occurring each year in Europe (in 2006 there were 45,822 new cases diagnosed in the UK: 45,508 woman and 314 men) and an estimated 182,460 in the USA (Office for National Statistics, 2006). These statistics underlie the intense activity in recent years to identify markers for prognosis and treatment of this disease. One area of scrutiny is the expression and distribution of cancer stem cell markers.

Stem cells are required for the maintenance of high cell turnover tissues where cells continually need to be replaced. They constitute a small population of relatively undifferentiated cells that express no differentiation markers of the tissue. They are slowly self-renewing with each cell division producing an average of one stem cell and one transit-amplifying cell. The transit-amplifying cell has limited proliferative potential, undergoing terminal differentiation to form the functioning cells of the tissue.

The 'tumor stem cell' theory suggests that a small percentage of cells in a tumor harbor intrinsic characteristics making them resistant to treatment. This could explain how patients with metastatic disease show clinical relapse several months after starting treatment due to the survival of a small group of cells with unique characteristics, including the ability to give rise to a new population of cells with a resistant phenotype.

Putative cancer stem and progenitor cells have been detected in a variety of epithelial cancers using markers that are associated with normal tissue stem cells but highly contextual (1). Previous studies have identified CD $44^{+} / \mathrm{CD} 24^{-}$breast cancer cells as candidate breast cancer stem cells (2), and CD133 (3,4). Park et al $(5)$ found that the expression of CD44 seemed to decrease with tumor progression in breast cancer and that $\mathrm{CD} 44^{+} / \mathrm{CD} 24^{-}$cells were most common in basal-like tumors. In a wide-range study of cell lines, expression of various stem cell marker (CD15, CD24, CD44, CD133, CD166, CD326) patterns was correlated with tumor entities such as basal breast cancer; other expression patterns occurred across different tumor types and were largely related to expression 
of a more mesenchymal phenotype in individual breast, lung, renal and melanoma cell lines (6).

Due to the renewed interest in the 'seed and soil' hypothesis of cancer first put forth by Paget $(7,8)$ in 1889 , there has been intense interest in 'cancer stem cell' markers. But as yet, few conclusions have been drawn as to their use as markers or prognostic indicators in solid human tumors. We chose 8 cancer stem cell markers in order to determine the distribution and expression pattern of these stem cell markers in human breast cancer. We found a significant association between loss of expression of CD24, CD34, CD44, CD49B, CD133 and PSCA and metastatic disease in patients with breast cancer. Such differential expression may play a part in breast cancer disease progression, and suggests that the current cancer stem cell theory may not hold true for all cancer types.

\section{Materials and methods}

Reagents and antibodies. Anti-CD44, -CD133, -CD49b and -PSCA antibodies were purchased from Pharmingen International (San Diego, CA, USA).

Tissue collection and preparation. Breast tissue samples (124 tumor and 33 matched adjacent tissues (Table I) (9) were collected and immediately frozen in liquid nitrogen before processing a portion of each sample for quantitative-PCR analysis, a portion for immunohistochemical analysis and a portion for routine histological examination. RNA was isolated from tissue samples using standard RNAzol procedures. For RT-PCR, cDNA was synthesized in a $20-\mu 1$ reaction mixture using $1 \mu \mathrm{g}$ RNA, as described in the protocol (ABgene Reverse Transcription System, Surrey, UK). The anonymized breast tissue samples were obtained according to the guidelines of the appropriate ethics committee (Bro Taf Health Authority 01/4303 and 01/4046). Informed patient consent was not applicable in this instance (as stated in the Human Tissue Act 2004, UK).

Quantitative-PCR. The q-PCR system used the Amplifluor ${ }^{\mathrm{TM}}$ UniPrimer $^{\mathrm{TM}}$ system (Intergen Company, Oxford, UK) and Thermo-Start $^{\circledR}$ (ABgene, Epsom, Surrey, UK). Specific primer pairs for CD24, CD29, CD34, CD44, CD49B, CD49F, CD133, PSCA and TSA-1 were designed by the authors using a Beacon Designer software (Biosoft International, Palo Alto, CA, USA) and manufactured by Invitrogen (Invitrogen Life Technologies, Paisley, Scotland, UK), each amplifying a region that spans at least 1 intron, generating an 100 base pair product from both the control plasmid and cDNA. The primers are as follows (5'-3'): CK-19QF1, caggtccgaggttactgac; CK19QZR1, actgaac ctgaccgtacacagtttctgccagtgtgtcttc; GAPDHF2, ctgagtacgtcgtg gagtc; GAPDHZR2, actgaacctgaccgtacacacagagatgatgaccctt tg; CD24F1, aactaatgccaaccaccaag; CD24ZR1, actgaacctgacc gtacataagagtagagatgcagaagag; CD29F1, cctgcettggtgtctgtg; CD29ZR1, actgaacctgaccgtacacctgtgtgcatgtgtctttc; CD34F1, tcagcaaagtggaagttat; CD34ZR1, actgaacctgaccgtacagtagtttgg gaatagctctg; CD44F1, accatggacaagttttggtggca; CD44ZR, act gaacctgaccgtacactgtagcgaccattttctc; CD49bF1, gcctgcagaaga atatggta; CD49bZR1, actgaacctgaccgtacatccagactgatgtccacac; CD49F1, gcgagccttcattgatgtg; CD49ZR1, actgaacetgaccgtacac tacagtctttgagggaaacac; CD133F1, gcaaatgtggaaaactgat; CD133ZR1, actgaacctgaccgtacattaaatagcttccagagaga;
PSCAF1, atgaaggctgtgctgctt; PSCAZR, actgaacctgaccgtacaag tcctcgttgctcacct; TSA1F1, cttgaaccagaagagcaatc; TSA1ZR1, actgaacctgaccgtacacactagcagacacagtcacg.

Using the $\mathrm{iCycler}^{\mathrm{iQ}} \mathrm{Q}^{\mathrm{TM}}$ system (Bio-Rad), which incorporates a gradient thermocycler and a 96-channel optical unit, the plasmid standards and breast cancer cDNA were simultaneously assayed in duplicated reactions using a standard HotStart q-PCR Master Mix. q-PCR conditions were as follows: enzyme activation at $95^{\circ} \mathrm{C}$ for $12 \mathrm{~min}$ for 1 cycle; followed by 60 cycles of denaturing at $95^{\circ} \mathrm{C}$ for $15 \mathrm{sec}$; annealing at $55^{\circ} \mathrm{C}$ for $40 \mathrm{sec}$; and extension at $72^{\circ} \mathrm{C}$ for $25 \mathrm{sec}$. Using purified plasmids as internal standards, the levels of cDNA (copies/50 ng RNA) in the breast cancer samples were calculated. q-PCR for $\beta$-actin was also performed on the same samples, to correct for any residual differences in the initial level of RNA in the specimens (in addition to spectrophotometry). Results were then normalized using cytokeratin-19 and GAPDH levels in the same tissues. The products of q-PCR were verified on agarose gels (data not shown). The data were then analyzed after a 10-year follow-up.

Immunohistochemistry. Cryostat sections of frozen tissue were cut at $6 \mu \mathrm{m}$, placed on SuperFrost Plus slides (LSL UK, Rochdale, UK), air dried and fixed in a 50:50 solution of alcohol:acetone. The sections were then air dried again and stored at $-20^{\circ} \mathrm{C}$ until used. Immediately before commencement of immunostaining, the sections were washed in buffer for $5 \mathrm{~min}$ and treated with horse serum for $20 \mathrm{~min}$ as a blocking agent to non-specific binding. Sections were stained using PSCA, CD44, CD133 and CD49b antibodies (Pharmingen International). Negative controls were used where necessary. Primary antibodies were used at a 1:100 dilution for $60 \mathrm{~min}$ and then washed in buffer. The secondary biotinylated antibody at a 1:100 dilution (Universal Secondary, Vectastain Elite ABC; Vector Laboratories Inc., Burlingame, CA, USA) was added (in horse serum/buffer solution) for $30 \mathrm{~min}$, followed by numerous washings. Avidin/biotin complex was added for $30 \mathrm{~min}$, again followed by washes. Diaminobenzidine was used as a chromogen to visualize the antibody/antigen complex. Sections were counterstained in Mayer's haematoxylin for 1 min, dehydrated, cleared and mounted in DPX. Following this, the sections were analyzed for staining intensity as previously described $(9,10)$.

Statistical analysis. Statistical analysis was performed by Minitab version 13.32 (Minitab Inc., State College, PA, USA) using a two-sample Student's t-test and the non-parametric Mann-Whitney confidence interval and test or Kruskal-Wallis, where appropriate. In addition, SPSS 12.01 was used to calculate the survival curves.

\section{Results}

Immunohistochemical staining of human breast tissues. Representative sections of tumor and normal human breast tissue sections are shown in Fig. 1A. IHC densitometry (Fig. 1B) revealed that there was a loss of expression of PSCA in tumor sections $(149.85 \pm 24.89)$ when compared to that in the background tissue $(222.85 \pm 9.88, \mathrm{n}=20, \mathrm{p}<0.0001)$. CD44 protein was strongly expressed in the background endo- 
Table I. Clinical characteristics of the patient samples.

$\begin{array}{cc}\text { Characteristics } & \begin{array}{c}\text { No. of patient } \\ \text { samples }\end{array}\end{array}$

\begin{tabular}{|c|c|}
\hline \multicolumn{2}{|l|}{ Tissue type } \\
\hline Background & 30 \\
\hline Tumor & 124 \\
\hline \multicolumn{2}{|l|}{ Grade } \\
\hline 1 & 24 \\
\hline 2 & 42 \\
\hline 3 & 58 \\
\hline \multicolumn{2}{|l|}{ NPI } \\
\hline 1 & 68 \\
\hline 2 & 38 \\
\hline 3 & 16 \\
\hline Unknown & 2 \\
\hline \multicolumn{2}{|l|}{ TNM status } \\
\hline 1 & 70 \\
\hline 2 & 40 \\
\hline 3 & 7 \\
\hline 4 & 4 \\
\hline Unknown & 3 \\
\hline \multicolumn{2}{|l|}{ Histology } \\
\hline Ductal & 94 \\
\hline Lobular & 14 \\
\hline Other & 16 \\
\hline \multicolumn{2}{|l|}{ Patient outcome } \\
\hline Alive and well & 85 \\
\hline Metastatic disease & 7 \\
\hline Death from breast cancer & 15 \\
\hline All poor outcomes & 27 \\
\hline
\end{tabular}

NPI, Nottingham Prognostic Indicator.

thelia and epithelia but was weaker and diffuse in tumor cells (134.5 \pm 29.89 and $187.9 \pm 21.28$ respectively, $\mathrm{p}<0.0001)$.

CD133 expression was lost in the tumor-associated endothelial cells with diffuse and weak staining in tumors when compared to the background $(194.9 \pm 35.76$ and $222.2 \pm 20.46$ respectively, $\mathrm{p}<0.006)$. Conversely, CD49b was strongly stained in the tumors and associated vessels and ducts but was weakly stained in the background epithelia, albeit that it was strongly stained in background ducts and vessels $(166.7 \pm 29.78$ and $211.75 \pm 15.32$ respectively, $\mathrm{p}<0.0001)$.

Quantitative PCR analysis of human breast tissues. q-PCR analysis of gene transcript levels (normalized using CK-19/GAPDH) further revealed the expression of the following stem cell markers: CD24, CD29, CD34, CD44, CD49b, CD49F, CD133, PSCA and TSA.

Stem cell marker expression and prognostic indicators. We compared the expression of all the stem cell markers with the diagnostic indicators Nottingham Prognostic Indicator (NPI), grade and TNM status (tumor nodal involvement). CD24, CD29, CD44 and CD133 were reduced with increasing NPI status (Table II), but did not reach statistical significance. CD49B, CD49F and PSCA showed no overall trend. Interestingly, CD34 was increased with increasing NPI (but did not reach significance). When looking at node-positive vs. node-negative tumors, CD133 was reduced in node-positive (node-negative $433 \pm 275$ vs. node-positive $147 \pm 102$ ) as

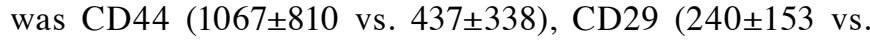
$67.8 \pm 22.3)$ and CD24 (1234 \pm 1075 vs. $435 \pm 239)$. However, node-positive tumors showed increased expression of CD49F $(702329 \pm 480290$ vs. $1175685 \pm 469885)$, CD $49 B(15.7 \pm 11.9$ vs. $31.5 \pm 17.9), \mathrm{CD} 34(2754 \pm 1422$ vs. $7676 \pm 4316)$ and PSCA $(11.78 \pm 5.91$ vs. $125 \pm 104)$.

When comparing grade 1 tumors with grade 2 and 3, it was found that all 8 stem cell markers were increased with increasing grade (Table III). However, only CD34 reached statistical significance $(\mathrm{p}=0.034)$.

Increasing TNM status was correlated with increased expression of CD29, CD34, CD44 and CD133 (Table II). CD24, CD49B, CD49B and PSCA were decreased with increasing TNM status. Again, this did not reach statistical significance.

Stem cell marker expression and estrogen receptor (ER) status. In ER-positive tumors, CD133 expression was increased ( $400 \pm 223$ vs. $452 \pm 370$, ER-negative vs. ER-positive, respec-

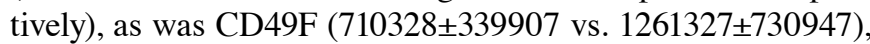
CD49B (15.5 \pm 12.5 vs. $38.6 \pm 20.2)$, CD34 (3521 \pm 1394 vs. $8448 \pm 6030)$, PSCA $(24.1 \pm 13.3$ vs. $150 \pm 148)$ and CD24 (311 \pm 161 vs. $2082 \pm 1595)$. Conversely, expression of CD44 was decreased in ER-positive tumors $(1122 \pm 683$ vs. $111 \pm 93.4)$ as was CD29 (189 \pm 119 vs. $143.5 \pm 72)$. Positive significance was not reached.

In the ER $\beta$-positive tumors, expression of CD133 was decreased $(518 \pm 242$ vs. $36.7 \pm 28.2$, ER $\beta$-negative vs. ER $\beta$-positive tumors, respectively), as was CD49F $(1016335 \pm 412127$ vs. $442909 \pm 317945), C D 49 B(27 \pm 13.3$ vs. $10.04 \pm 9.96), \mathrm{CD} 44$ ( $942 \pm 550$ vs. $18.1 \pm 16.1, \mathrm{p}=0.097), \mathrm{CD} 29$ (203 \pm 102 vs. $53.6 \pm 29.1)$ and CD24 (1108 \pm 706 vs. $187 \pm 160)$. In comparison, $\mathrm{CD} 34$ was increased in ER $\beta$-positive tumors $(4568 \pm 2605$ vs. $6974 \pm 4110, E R \beta$-negative vs. ER $\beta$-positive tumors, respectively), as was the level of PSCA (19.7 \pm 11 vs. $226 \pm 222)$.

Stem cell marker expression and patient clinical outcome. CD44, CD34, CD49B and PSCA were reduced in tumors of patients with poor outcome (metastatic disease and death from breast cancer) (Fig. 2A-D). CD133 was significantly reduced in tumors from patients with metastatic disease $(1.66 \pm 1.02)$ when compared with those remaining alive and well after the 10-year follow-up (553 $\pm 261, \mathrm{p}=0.038$ ) (Fig. 2E). CD24 was dramatically reduced in metastatic disease, but not in the patients who had died from breast cancer (Fig. 2F). Conversely, CD29 was slightly elevated in metastatic disease, but reduced in those patients who had died from breast cancer (Fig. 2G). The only marker to show increased expression in all poor outcomes, compared to those patients who remained alive and well was CD49F (Fig. 2H). 

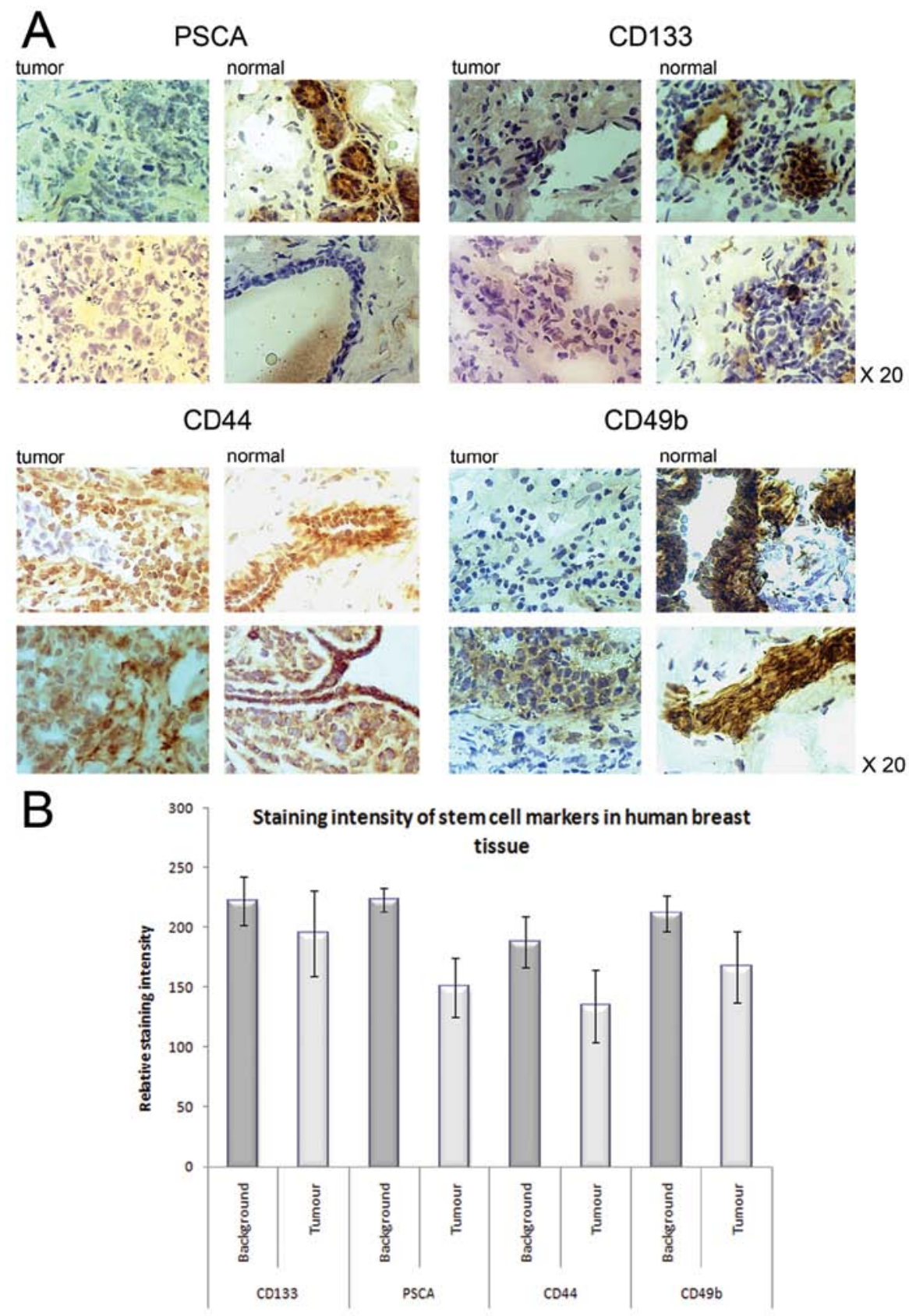

Figure 1. (A) Immunohistochemical analysis of human breast tumor and normal tissue sections stained for PSCA, CD133, CD44 and CD49b. (B) Quantification of positive staining in the sections. All 4 markers showed reduced expression in the tumor sections.

Stem cell marker expression and metastatic disease to bone. Since tissues from patients with metastatic disease had a correlation with the stem cell markers in relation to patient outcome, we decided to dissect the metastatic data further to consider the expression of these markers. CD133 was again markedly reduced in metastasis to bone (alive and well, 553 \pm 261 ; bone metastasis, $110 \pm 91 ; \mathrm{p}=0.067$ ). The expression of CD49B was also significantly reduced in bone metastasis and in bone metastasis leading to death (alive and well, $31.15 \pm 15$; bone metastasis, $0.00054 \pm 0.0005$; $\mathrm{p}=0.039$; bone metastasis and death, $0.75 \pm 0.7 ; \mathrm{p}=0.044)$. CD44 was also reduce in bone metastasis, but did not reach significance (alive and well, 968 \pm 599 ; bone metastasis, $2.58 \pm 1$.6). There was also a reduction in expression of PSCA, CD29 and CD24 in bone metastasis (PSCA, $20.5 \pm 12$ vs. $0.06 \pm 0.05 ; \mathrm{p}=0.09$; $\mathrm{CD} 29$, $200 \pm 111$ vs. $63 \pm 50 ; \mathrm{CD} 24,1021 \pm 761$ vs. $50 \pm 44$, alive and well vs. bone metastasis, respectively). The results for CD29 were in contrast to the levels found in metastatic disease, which were increased. Moreover, CD34 was increased in bone metastasis, which was in contrast to metastatic disease overall (alive and well, $4080 \pm 1541$ vs. bone metastasis, $25166 \pm 23318$ vs. all metastatic disease, $700 \pm 701$ ) but this did not reach significance. Again, CF49F was increased in tissues from patients with bone metastasis (alive and well, $704108 \pm 309573$ vs. bone metastasis, $3109444 \pm 3000851$ ) but did not reach significance.

Stem cell marker expression and ER status in patient survival. We further dissected the results obtained for survival in 


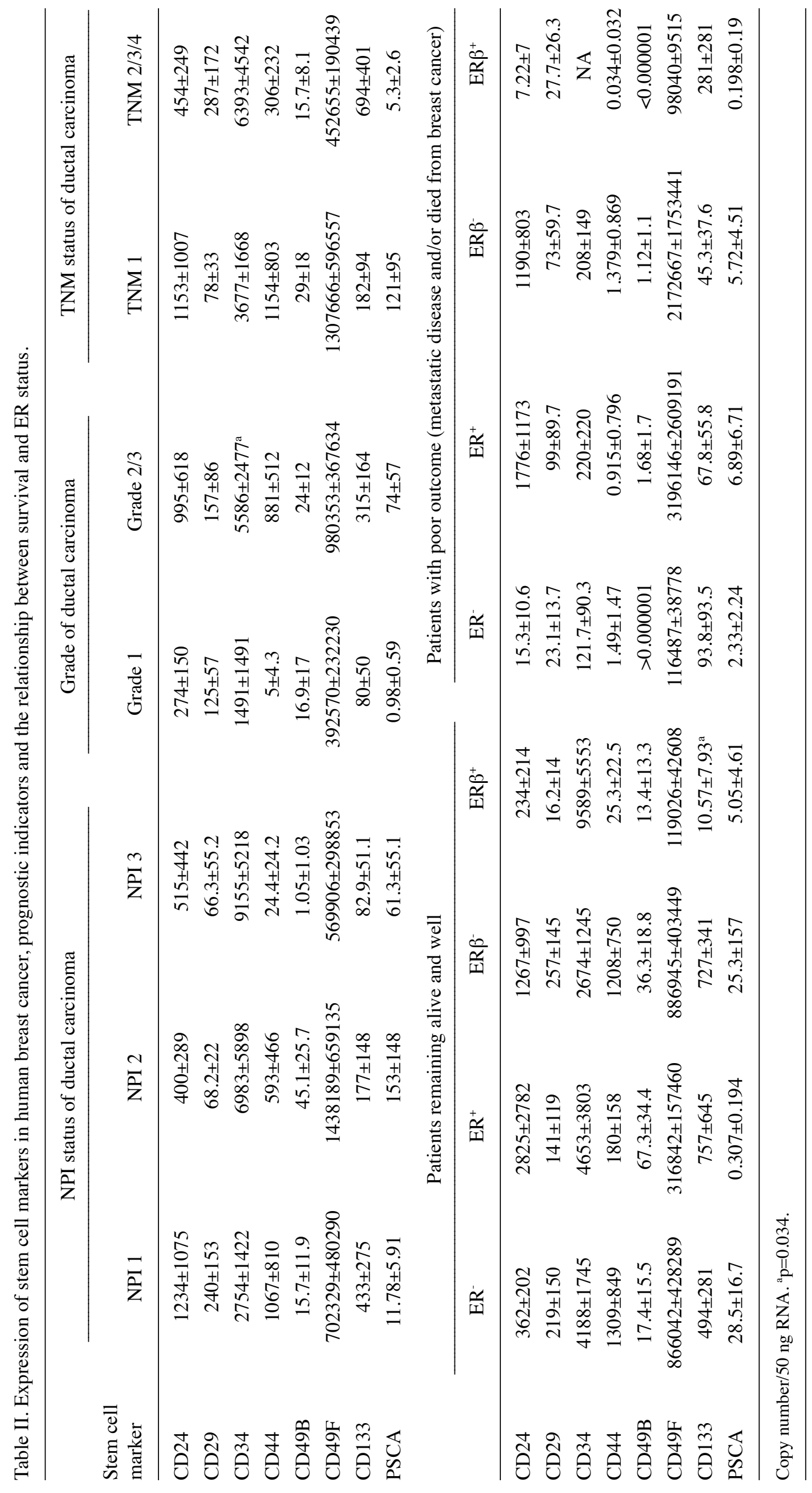


regard to ER status. Overall, in patients who had remained alive and well, as shown in Table II, CD24, CD34, CD49B, CD49F and CD133 were increased in ER-positive tumors, with CD29, CD44 and PSCA being reduced. This increase was significant for CD133 ( $\mathrm{p}=0.041)$. CD24, CD29, Cd44, CD49B, CD133 and PSCA were reduced in ER $\beta$-positive tumors, with CD34 and CD49F being decreased. In patients who had poor outcome (i.e. had metastatic disease or died from breast cancer), CD24, CD29, CD34, CD49B, CD49F and PSCA were increased in ER-negative tumors, whereas CD44 and CD133 were decreased. CD34, CD29, CD44, CD49F, PSCA and CD49B were reduced in ER $\beta$-positive tumors (data not available for CD34), with CD133 being the only marker to be increased.

Stem cell marker expression and ductal carcinoma. When tumor type was considered, the data showed that CD44, CD29 and CD34 were elevated in ductal carcinoma in comparison to other tumor types (CD44 ductal, $8719 \pm 536$ vs. other types, 13.1 \pm 12.9 ; CD29 ductal, $173 \pm 95.6$ vs. other types, $148 \pm 121$; CD34 ductal, 6054 \pm 2573 vs. other types, 101 $\pm 79 ; \mathrm{p}=0.023$ ). CD133, CD49B, PSCA and CD49F were reduced in ductal carcinoma (CD133 ductal, $186 \pm 83$ vs. other types, $1162 \pm 757$;

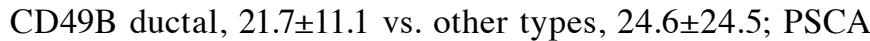
ductal, 20.4 \pm 10.7 vs. other types, $212 \pm 211$; CD49F ductal, $1080198 \pm 407865$ vs. other types, 221874 \pm 113113 ; $\mathrm{p}=0.046$ ). We then further investigated how these stem cell markers were expressed in ductal carcinoma.

Stem cell marker expression in ductal carcinoma and prognostic indicators. When considering ductal carcinomas alone, it was noted that with increasing NPI status, there was a corresponding increase in expression of CD34 and PSCA (Table III). Overall, the other stem cell markers showed reduced expression with increasing NPI, which was significant for CD49F ( $\mathrm{p}=0.012$ ). CD34 was also increased with increasing grade as was CD44, CD49F, CD133 and PSCA. There was a decrease in expression of CD24 $(\mathrm{p}=0.02), \mathrm{CD} 29$ and CD49B with increasing grade of ductal carcinomas. Only CD34 and CD29 showed an elevated expression in high (2-4) TNM status (Table III). CD49F was significantly decreased $(\mathrm{p}=0.047)$.

Stem cell marker expression in ductal carcinoma and patient outcome. In the ductal carcinomas, CD24 expression was reduced in metastatic disease and in those patients who had died from breast cancer (Fig. 3A). Similar results were observed for CD29, CD34, CD49B, CD133 ( $\mathrm{p}=0.036$ ) and PSCA (Fig. 3B-F). In contrast, expression of CD44 showed some increase in metastatic disease, although levels were reduced in patients who had died from ductal carcinoma (Fig. 3G). Moreover, CD49F was markedly increased in metastatic disease and all poor outcomes (Fig. 3H). This distribution was similar to that found in breast cancer overall (discussed above), apart from CD44. When looking more closely at bone metastasis, it was found that the expression of CD24, CD29, CD34, CD44 and CD133 in bone metastasis in ductal carcinoma was not different to that in bone metastasis overall (Fig. 4A-E). However, levels of CD49F were increased in bone metastasis in ductal carcinoma and reduced CD49B 


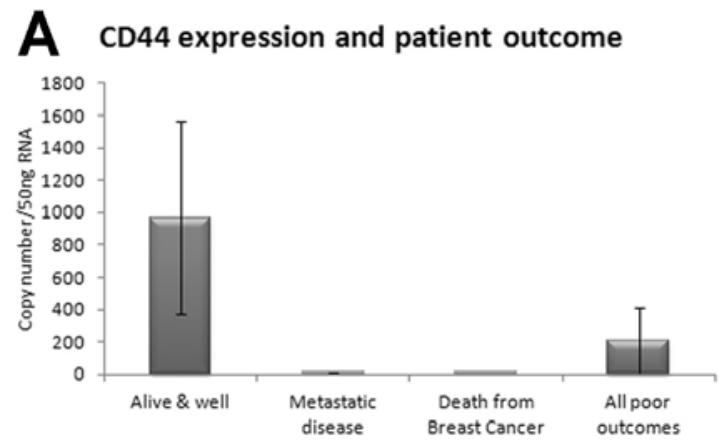

C CD49B expression and patient outcome

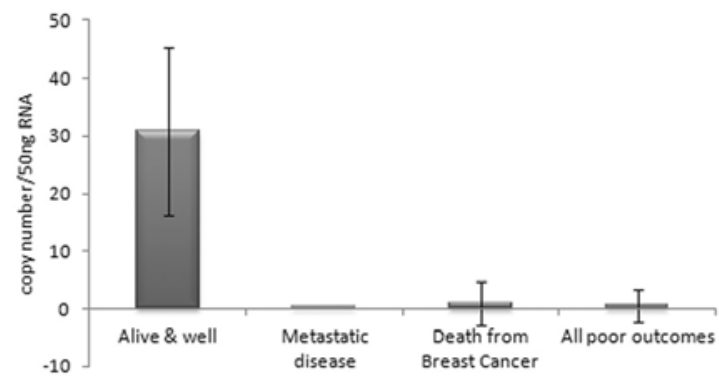

E CD133 expression and patient outcome

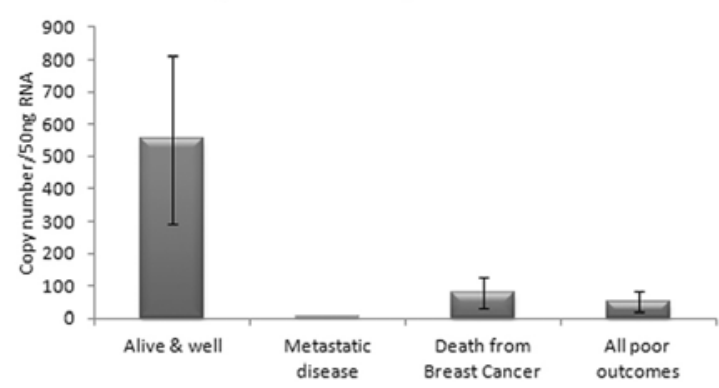

G CD29 expression and patient outcome

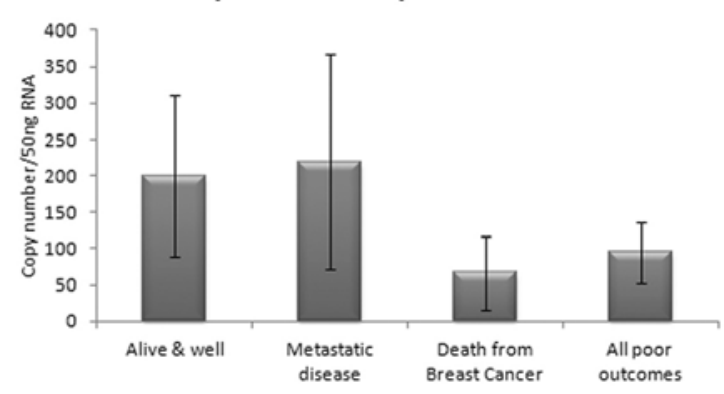

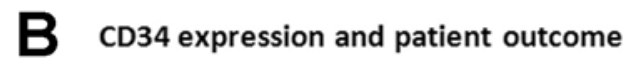

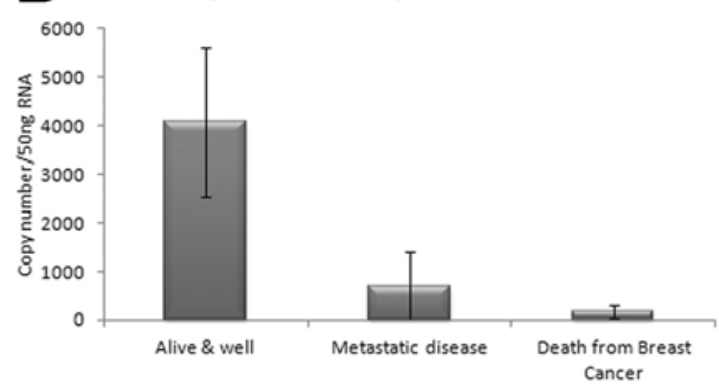

D PSCA expression and patient outcome

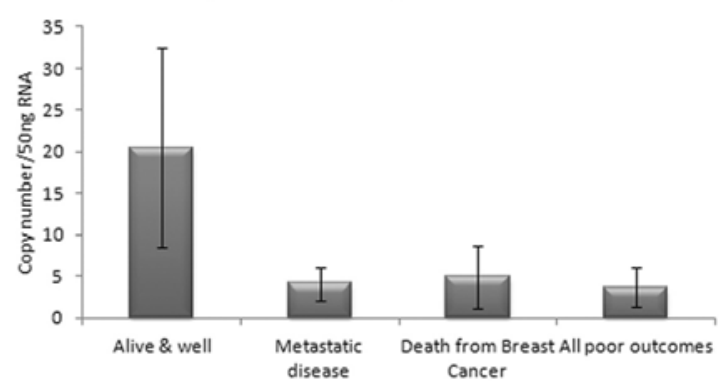

F CD24 expression and patient outcome

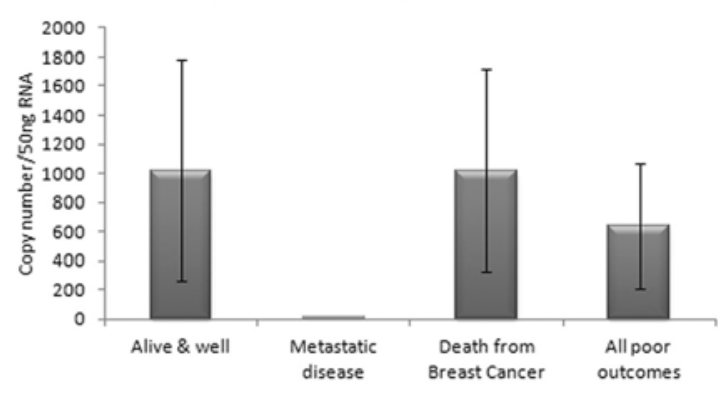

H CD49F expression and patient outcome

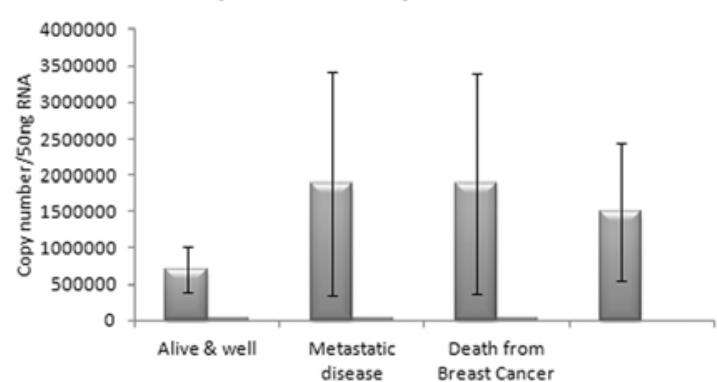

Figure 2. Analysis of cancer stem cell markers in human breast cancer and patient outcome using q-PCR.

expression was noted (Fig. 4F and G). Levels for PSCA were unavailable.

Stem cell markers and survival. A long-term survival curve incorporating all of the markers was calculated using Kaplan-Meier survival curves (Fig. 4H). Patients with high levels of combined transcripts had a significantly shorter survival than patients with low levels $(\mathrm{p}=0.006)$; high mean survival of 109.55 months (90.666-128.435 months, 95\% CI) vs. low mean survival of 143.106 months (134.415-151.797 months, 95\% CI) with cut-offs as previously determined (9).

\section{Discussion}

The most widely accepted model for metastasis is the 'seed and soil' hypothesis postulated by Paget (7). He suggested that 


\section{A Expression of CD24 in ductal carcinoma and patient outcome}

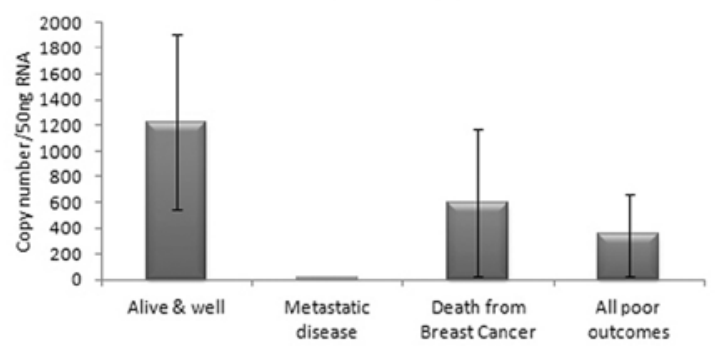

C Expression of $\mathrm{CD} 34$ in ductal carcinoma and patient outcome

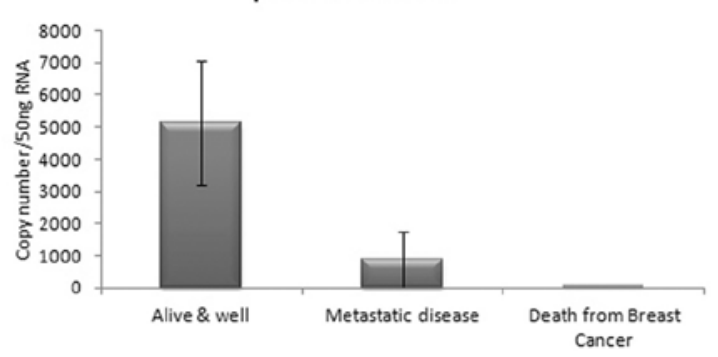

E Expression of $\mathrm{CD} 133$ in ductal carcinoma and patient outcome

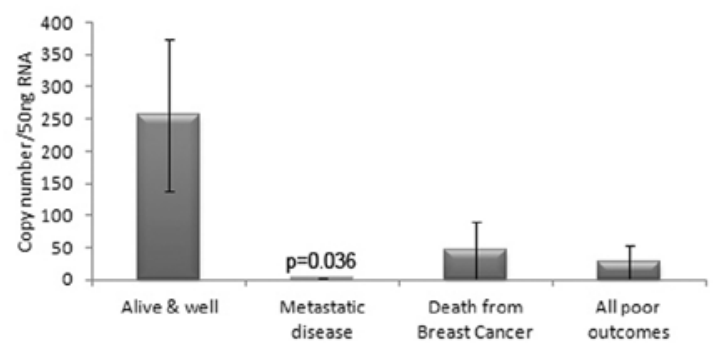

G Expression of $\mathrm{CD} 44$ in ductal carcinoma and patient outcome

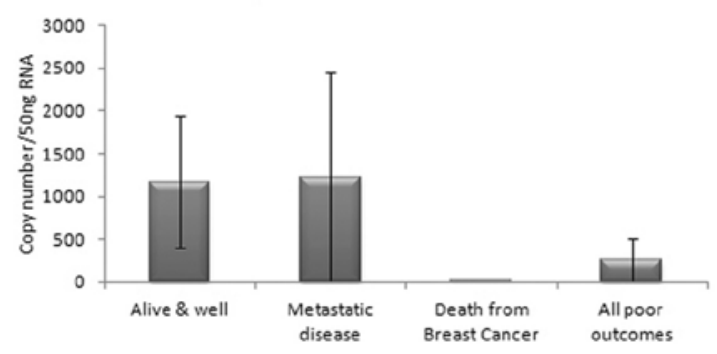

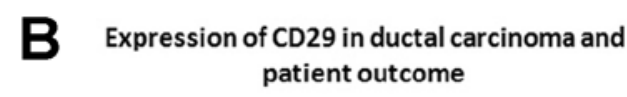

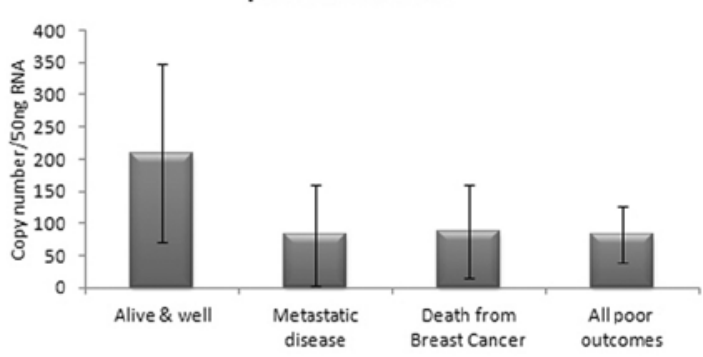

D Expression of CD49B in ductal carcinoma and patient outcome
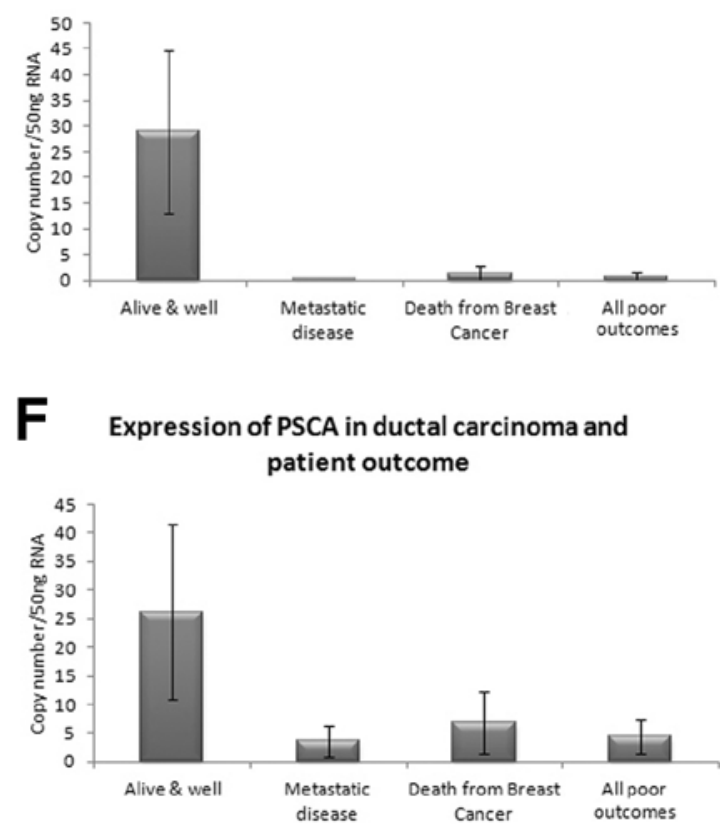

H Expression of CD49F in ductal carcinoma and patient outcome

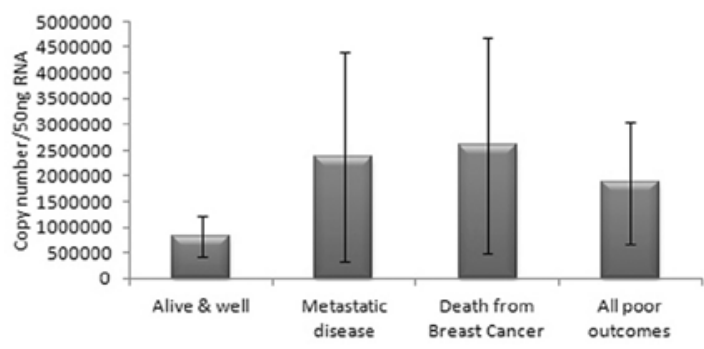

Figure 3. Analysis of the correlation between cancer stem cell markers in ductal carcinoma and patient outcome using q-PCR.

malignant tumor cells are shed from the primary tumor and disseminated in the entire body although they will metastasize when the shed (disseminated tumor cells) and soil (secondary organ) are compatible. Subsequently, knowledge in this area has expanded significantly. However, the mechanisms underlying the entire process are still unclear, and currently available therapies are mainly palliative. The tumor stem cell hypothesis suggests that there exists within cancers, a subset of cancer cells that are responsible for tumor recurrence following chemotherapy and are causative of metastasis. Studies using human cell lines and human tissues suggest that a pattern of cell surface and functional markers define these cancer stem cells (6). Our study is one of the first to determine the distribution and expression pattern of a number of stem cell markers in human breast cancer.

As attractive as the cancer stem cell hypothesis sounds, especially when applied to tumors that respond poorly to current treatments, it has been argued that the proposal of a stem-like cell that initiates and drives solid tissue cancer growth and is responsible for therapeutic failure is far from proven (11). 


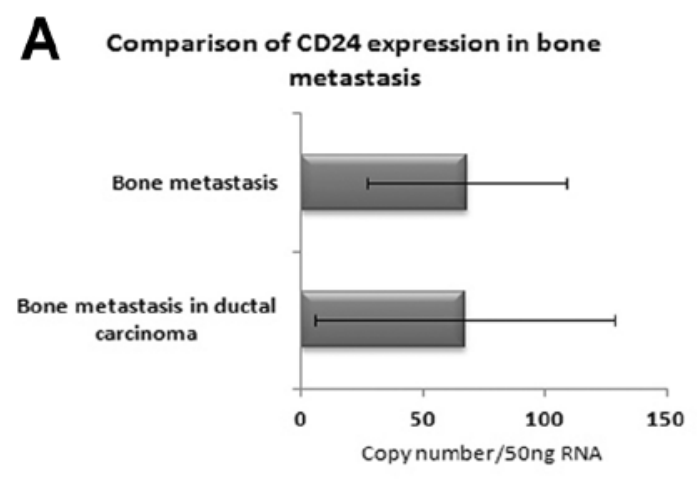

C Comparisonof $\mathrm{CD} 34$ expression in bone metastasis

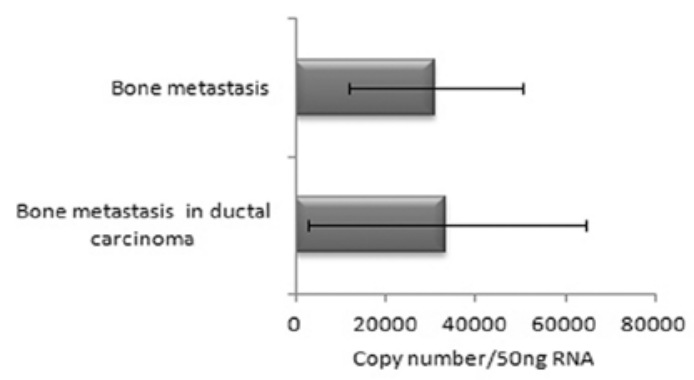

E Comparison of CD133 expression in bone metastasis

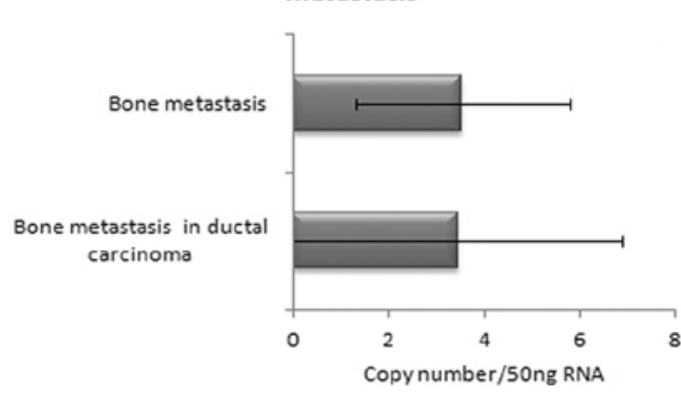

G Comparison of CD49B expression in bone

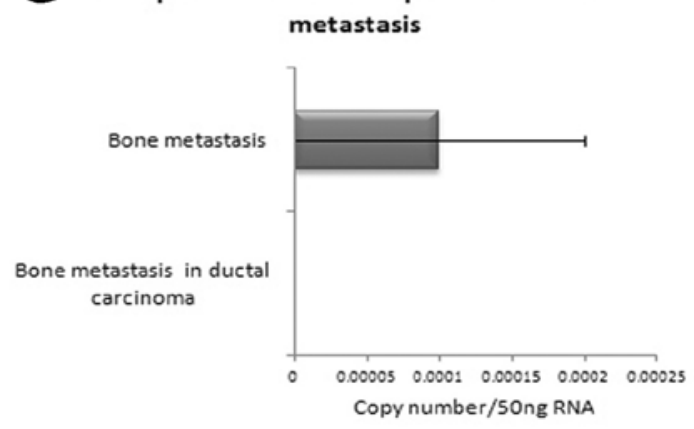

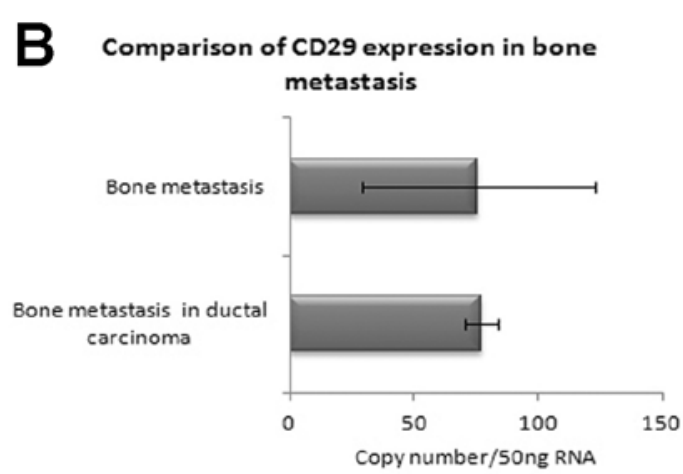
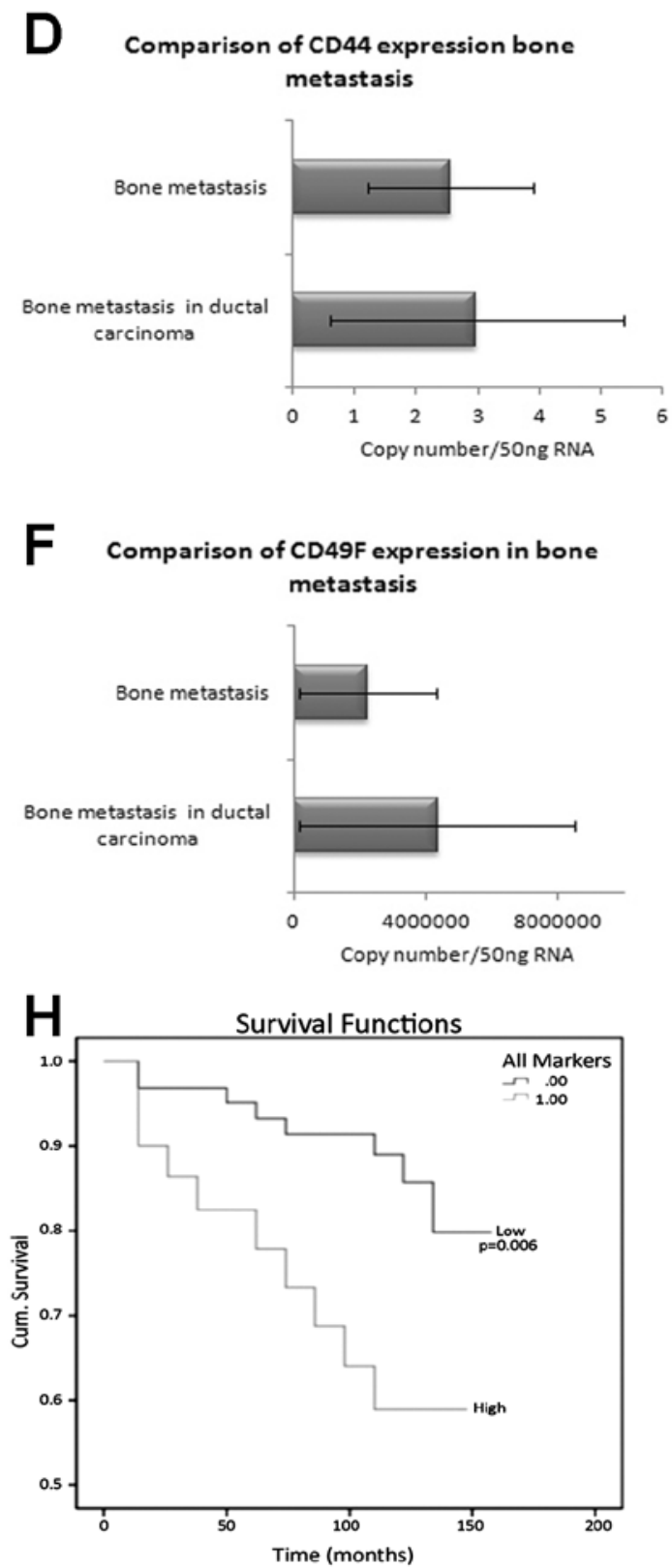

Figure 4. Correlation of expression of cancer stem cell marker in bone metastasis in all breast tumors and in ductal carcinomas and cumulative survival analysis, as analyzed using q-PCR.

In the present study, we demonstrated that overall, there was a reduction in expression of CD24, CD29, CD44 and CD133 with increasing NPI, an increase in all markers with increasing grade and an increase in expression of CD29, CD34, CD44 and CD133 with increasing TNM status. Moreover, the majority of cancer stem markers were reduced with metastatic 
disease and poor prognosis overall. Only CD29 was increased with metastatic disease, and patients who had died from breast cancer had increased levels of CD24.

Although a broad range of pathological subtypes of invasive breast cancer have been identified, the most commonly used classification of invasive breast cancers divides them into ductal and lobular types. Invasive ductal carcinoma represents the largest group, accounting for up to $80 \%$ of invasive cancers (12). When comparing ductal carcinoma and other cancer types, we found that CD34, CD29 and CD44 were elevated. In the patients with ductal carcinoma, we found that while for the most part the distribution of cancer stem cell markers mirrored that of breast cancer overall, CD44 was increased in metastatic disease and CD49F was increased in all poor outcomes.

Breast carcinomas have been reported to contain a subpopulation of $\mathrm{CD}^{4} 4^{+} / \mathrm{CD} 24^{-}$tumor cells with stem cell-like properties. In fact, the majority of studies to date have tended to concentrate on $\mathrm{CD} 44^{+} / \mathrm{CD} 24^{-}(13-17)$ and there have been few that have examined a larger range of markers in the same cohort. Giatromanolaki et al (18) investigated the significance of these 2 molecules in connection with tumor aggression and prognosis. The phenotypic profile of 139 breast carcinomas was investigated in paraffin sections using markers previously associated with stem cell-like properties (CD44, CD24), the 'triple-state' (ER, PR, c-erb-B2) and angiogenesis (CD31). The authors concluded that assessment of the CD44/CD24 status may reveal distinct subgroups of breast cancer patients with different clinical behavior, and that CD44 targeting was an attractive therapeutic alternative for breast cancer patients and that the strong association between the CD44\%CD24- phenotype and prognosis required further investigation.

Such studies are plainly in contrast to the study presented here. However, Ahmed et al (19) suggested that the $\mathrm{CD} 44^{-} / \mathrm{CD} 24^{+}$phenotype is a poor prognostic marker in early invasive breast cancer. Breast cancer cells with high CD44 and low or absent CD24 are reported to have stem cell features. The authors stated that CD24 and CD44 expression can individually yield prognostic data in breast cancer, but importantly, when both markers are considered, CD $44^{+} / \mathrm{CD} 24$ was associated with the best prognosis, while CD $44^{-} / \mathrm{CD} 24^{+}$ was associated with the worst prognosis. This shows that the relationship between basic cell biology and clinical behavior is not always straightforward and warrants further investigation of the true clinical impact of breast cancer stem cells (19). It has been demonstrated that individual CD44 isoforms can be associated with different breast cancer subtypes and clinical markers such as HER2, ER and PgR, which suggests involvement of CD44 splice variants in specific oncogenic signaling pathways (20). Efforts therefore, to link CD44 to cancer stem cells and tumor progression should consider the expression of various CD44 isoforms. Moreover, in a recent study by Guler et al (21), the occurrence of CD24/44+ and CD24 ${ }^{+} / 44^{-}$cells in primary tumors did not differ in primary vs. matched lymph node or distant and locoregional metastatic lesions. Thus, the frequency of CD24 $/ 44^{+}$cells does not differ in metastases relative to the primary breast cancer but differs in regards to tumor stage and subtype.

Approximately $70 \%$ of patients with breast cancer have bone metastases. They are associated with poor prognosis and the available treatment options are very limited (22). Bone metastasis usually presents with severe pain, and these symptoms are usually noted in the femur and pelvic region. Of the 2 types of breast cancer bone metastasis, osteolytic lesions are the most common form and cause destruction of the bone whereas osteoblastic lesions, which are less common, cause new bone formation. Most patients have components of both bone resorption and bone formation. When we examined our data more intensely in order to determine any differences in bone metastasis, we discovered that there was, overall, very little difference in the expression of the cancer stem cell markers in all patients with bone metastasis, compared to patients with ductal carcinoma. CD49F, however, was increased in bone metastasis in ductal carcinoma, whereas CD48B was completely lost.

The metadata analysis conducted by Zhou et al (16) lent support to the cancer stem cell hypothesis by showing a significant correlation between cancer stem cells and common clinical parameters, such as ER, PR, HER2 and tumor grade. Putative stem cell markers, particularly ALDH1, were significantly associated with worse survival based on currently obtained data. It was suggested by the authors that these markers should be further evaluated for their potential use in the identification of breast cancer stem cells in clinical practice.

In conclusion, we demonstrated that there is a significant association between loss of expression of certain stem cell markers and metastatic disease in patients with breast cancer. Such differential expression may play a part in breast cancer disease progression, and suggests that the current stem cell theory may not entirely hold true for all cancer types and that investigations into wider types of solid tumors and covering a broader range of 'stem cell markers' are required.

\section{Acknowledgements}

We would like to thank Cancer Research Wales (CRW) for sponsoring the present study and Dr Anthony Douglas-Jones for his invaluable help and assistance with patient samples.

\section{References}

1. Donnenberg VS, Donnenberg AD, Zimmerlin L, Landreneau RJ, Bhargava R, Wetzel RA, Basse P and Brufsky AM: Localization of CD44 and CD90-positive cells to the invasive front of breast tumors. Cytometry B Clin Cytom 78: 287-301, 2010.

2. Ginestier C, Korkaya H, Dontu G, Birnbaum D, Wicha MS and Charafe-Jauffret EI: The cancer stem cell: the breast cancer driver. Med Sci 23: 1133-1139, 2007 (In French).

3. Wright MH, Calcagno AM, Salcido CD, Carlson MD, Ambudkar SV and Varticovski L: Brcal breast tumors contain distinct $\mathrm{CD} 44^{+} / \mathrm{CD} 24^{-}$and $\mathrm{CD} 133^{+}$cells with cancer stem cell characteristics. Breast Cancer Res 10: R10, 2008.

4. Xiao Y, Ye Y, Yearsley K, Jones S and Barsky SH: The lymphovascular embolus of inflammatory breast cancer expresses a stem cell-like phenotype. Am J Pathol 173: 561-574, 2008.

5. Park SY, Lee HE, Li H, Shipitsin M, Gelman R and Polyak K: Heterogeneity for stem cell-related markers according to tumor subtype and histologic stage in breast cancer. Clin Cancer Res 16: 876-887, 2010.

6. Stuelten CH, Mertins SD, Busch JI, Gowens M, Scudiero DA, Burkett MW, Hite KM, Alley M, Hollingshead M, Shoemaker RH and Niederhuber JE: Complex display of putative tumor stem cell markers in the NCI60 tumor cell line panel. Stem Cells 28: 649-660, 2010 
7. Paget S: The distribution of secondary growths of the breast. Lancet 1: 571-573, 1889.

8. Lin EH, Jiang Y, Deng Y, Lapsiwala R, Lin T and Blau CA: Cancer stem cells, endothelial progenitors, and mesenchymal stem cells: 'seed and soil' theory revisited. Gastrointest Cancer Res 2: 169-174, 2008.

9. Martin TA, Watkins G, Mansel RE and Jiang WG: Loss of tight junction plaque molecules in breast cancer tissues is associated with a poor prognosis in patients with breast cancer. Eur J Cancer 40: 2717-2725, 2004.

10. Martin TA, Pereira G, Watkins G, Mansel RE and Jiang WG: $\mathrm{N}$-WASP is a putative tumour suppressor in breast cancer cells, in vitro and in vivo, and is associated with clinical outcome in patients with breast cancer. Clin Exp Metastasis 25: 97-108, 2008.

11. Rahman M, Deleyrolle L, Vedam-Mai V, Azari H, Abd-ElBarr M and Reynolds BA: The cancer stem cell hypothesis: failures and pitfalls. Neurosurgery 68: 531-545, 2011.

12. Weigelt B, Horlings HM, Kreike B, Hayes MM, Hauptmann M, Wessels LF, de Jong D, Van de Vijver MJ, Van't Veer LJ and Peterse JL: Refinement of breast cancer classification by molecular characterization of histological special types. J Pathol 216: 141-150, 2008.

13. Bhat-Nakshatri P, Appaiah H, Ballas C, Pick-Franke P Goulet R Jr, Badve S, Srour EF and Nakshatri H: SLUG/SNAI2 and tumor necrosis factor generate breast cells with $\mathrm{CD} 44^{+} / \mathrm{CD} 24$ phenotype. BMC Cancer 10: 411, 2010.

14. Cipak A, Mrakovcic L, Ciz M, Lojek A, Mihaylova B, Goshev I, Jaganjac M, Cindric M, Sitic S, Margaritoni M, Waeg G, Balic M and Zarkovic N: Growth suppression of human breast carcinoma stem cells by lipid peroxidation product 4-hydroxy-2-nonenal and hydroxyl radical-modified collagen. Acta Biochim Pol 57: 165-171, 2010.

15. Oliveras-Ferraros C, Vazquez-Martin A, Martin-Castillo B, Martin-Castillo B, Cufí S, Del Barco S, Lopez-Bonet E, Brunet J and Menendez JA: Dynamic emergence of the mesenchymal $\mathrm{CD} 44^{\text {pos }} \mathrm{CD} 24^{\text {neg/low }}$ phenotype in HER2-gene amplified breast cancer cells with de novo resistance to trastuzumab (Herceptin). Biochem Biophys Res Commun 397: 27-33, 2010.
16. Zhou L, Jiang Y, Yan T, Di G, Shen Z, Shao Z and Lu J: The prognostic role of cancer stem cells in breast cancer: a metaanalysis of published literatures. Breast Cancer Res Treat 122: 795-801, 2010.

17. Fillmore $C$ and Kuperwasser C: Human breast cancer stem cell markers CD44 and CD24: enriching for cells with functional properties in mice or in man? Breast Cancer Res 9: 303, 2007.

18. Giatromanolaki A, Sivridis E, Fiska A and Koukourakis MI: The CD $44^{+} / \mathrm{CD} 24^{-}$phenotype relates to 'triple-negative' state and unfavorable prognosis in breast cancer patients. Med Oncol 28: 745-752, 2011.

19. Ahmed MA, Aleskandarany MA, Rakha EA, Moustafa RZ, Benhasouna A, Nolan C, Green AR, Ilyas M and Ellis IO: A $\mathrm{CD} 44 \% \mathrm{CD} 24^{+}$phenotype is a poor prognostic marker in early invasive breast cancer. Breast Cancer Res Treat 133: 979-995, 2012.

20. Olsson E, Honeth G, Bendahl PO, Saal LH, GruvbergerSaal S, Ringnér M, Vallon-Christersson J, Jönsson G, Holm K, Lövgren K, Fernö M, Grabau D, Borg A and Hegardt C: CD44 isoforms are heterogeneously expressed in breast cancer and correlate with tumor subtypes and cancer stem cell markers. BMC Cancer 11: 418, 2011.

21. Guler G, Balci S, Costinean S, Ussakli CH, Irkkan C, Suren D, Sari E, Altundag K, Ozisik Y, Jones S, Bacher J, Shapiro CL and Huebner K: Stem cell-related markers in primary breast cancers and associated metastatic lesions. Mod Pathol 25: 949-955, 2012.

22. Akhtari M, Mansuri J, Newman KA, Guise TM and Seth P: Biology of breast cancer bone metastasis. Cancer Biol Ther 7: 3-9, 2008. 